\title{
ARIANE 5 SOLID ROCKET BOOSTER DYNAMIC BEHAVIOR WITH RESPECT TO PRESSURE OSCILLATIONS
}

G. Durin ${ }^{1}$, F. Bouvier ${ }^{1}$, G. Mastrangelo ${ }^{2}$, and E. Robert ${ }^{3}$

${ }^{1}$ Snecma Propulsion Solide, SAFRAN Group Les cinq chemins, Le Haillan Cedex 33187, France

${ }^{2}$ Europropulsion

Rue Salomon de Rothschild, Suresnes 92150, France

${ }^{3} \mathrm{CNES}$

Rond Point de L'Espace, Evry Cedex 91923, France

\begin{abstract}
Numerical simulations are performed to simulate the dynamic behavior of the Ariane 5 Solid Rocket Booster (SRB) excited by internal pressure oscillations. These pressure oscillations have sliding frequencies close to acoustic natural frequencies and changing amplitudes. Because the modal behavior of the booster is also continuously changing during the flight due to propellant burning, predictions with finite-element (FE) modeling (modal analyses, harmonic and transient responses) are necessary to predict and understand the dynamic behavior of the booster.
\end{abstract}

\section{INTRODUCTION}

\subsection{Pressure Oscillations}

In SRB, pressure oscillations result from instabilities characterized by the presence of vortices in the flow. Some configurations of the combustion chamber are favorable for the development of these instabilities:

- the combustion surface of the propellant: the longer the segments, the stronger the vortices;

- the inhibitors located on the top of each propellant surface: these rubber inhibitors represent an obstacle to the upstream flow and induce a shear layer between two different gas flows; and

This is an Open Access article distributed under the terms of the Creative Commons Attribution-Noncommercial License 3.0, which permits unrestricted use, distribution, and reproduction in any noncommercial medium, provided the original work is properly cited. 
- the geometry of propellant loading: angles between burning surfaces also generate shear layers in the flow.

Efforts can be done to reduce pressure oscillations, but it is not possible to remove them because the conditions that generate instabilities cannot disappear.

The SRB pressure oscillations occur mainly during the second half of combustion in some specific ranges of frequency: some instabilities are continuously generated at any frequency, but the only ones which can be observed and which are critical have a frequency close to the chamber acoustic natural frequencies (otherwise, the phenomenon cannot be amplified and sustained). Because the geometry of the chamber is modified during combustion, both frequencies and

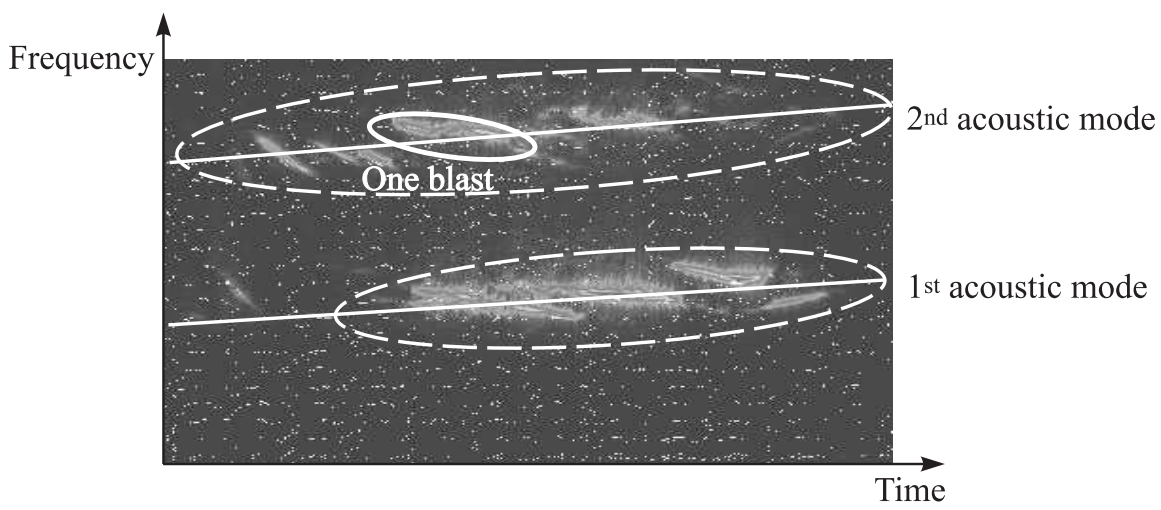

Figure 1 Sliding spectrum on SRB pressure measurement

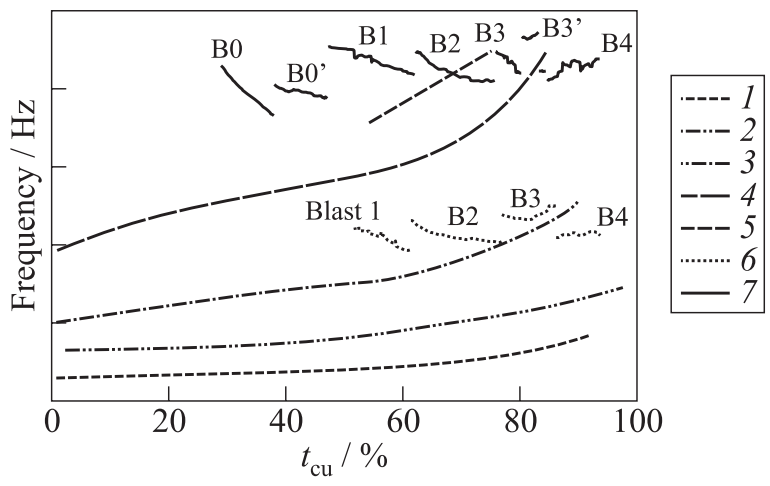

Figure 2 Structural natural frequencies in test-bench configuration (1-4 - modes 14 (bending) and 5 - mode 5 (longitudinal elongation)) and pressure oscillations frequencies: 6 and $7-1$ st and 2 nd AM, respectively 
amplitudes of pressure oscillations are evolutionary as shown in Fig 1. Pressure oscillations are organized in a succession of blasts, linked together as frequency waterfalls around either the first or the second acoustic frequency of the cavity.

Some measurement treatments allow restoring both frequency and amplitude evolutions for the different blasts of the 1st or 2nd acoustic mode (AM) pressure oscillations (frequency evolution shown in Fig. 2).

\subsection{Solid Rocket Booster Modal Behavior and Resonance Risks}

The structural eigenmodes of the SRB depend on both mass and stiffness (including boundary conditions) of the booster. Assuming that the stiffness of the booster does not change during combustion (stiffness of the case is not modified), natural frequencies logically increase due to the loss of propellant mass. The frequencies of the first structural eigenmodes are in the same range as the 1st and 2nd acoustic frequencies, which can produce a high dynamic amplification of pressure excitation in case of resonance. Most of these excitable eigenmodes are bending modes in the yaw plane, mainly, because of the symmetry conditions.

Figure 2 presents the evolution of frequencies of pressure oscillations (1st and 2nd AM) and of structural eigenmodes (bending and elongation). It clearly appears that SRB dynamic conditions of the response to pressure oscillations change with combustion time and excitation frequency. It can be even noted that some resonance conditions exist in the test-bench configuration between $70 \%$ and $80 \%$ of effective combustion time $\left(t_{\mathrm{cu}}\right)$ for different blasts of the 1 st and 2nd AM:

- around $77 \%$ and $82 \%$, concerning the 2 nd and 3rd blasts of the 1 st AM ("B2" and "B3") and the structural mode No.3: the SRB is excited in almost resonance conditions; and

- around $69 \%$, concerning the 2nd blast of the 2nd AM ("B2") and the structural mode No. 5: the SRB is excited in pure resonance conditions.

These resonance conditions can be problematic for the SRB dynamic behavior: it is necessary to simulate it finely, firstly, for correct understanding of experimental observations and measurements and, secondly, to offer reliable predictions.

\section{FINITE-ELEMENT SIMULATIONS}

\subsection{Available Finite-Element Models}

In the frame of SRB development, Snecma Propulsion Solide (Safran Group) has developed the models of this motor for the account of Europropulsion and CNES. 


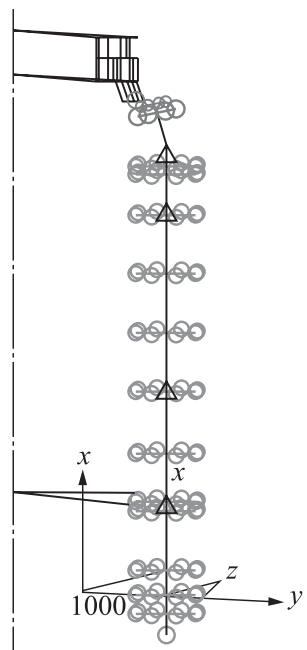

(a)

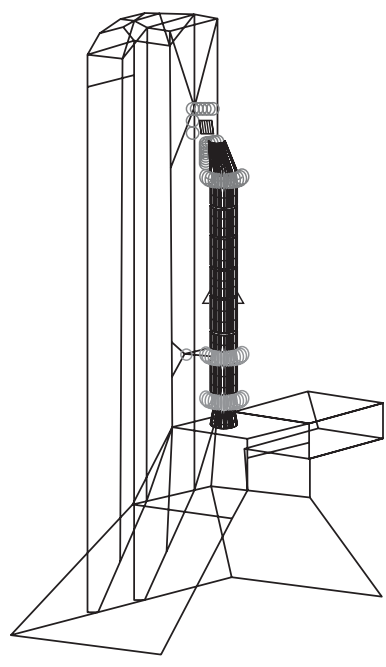

(b)

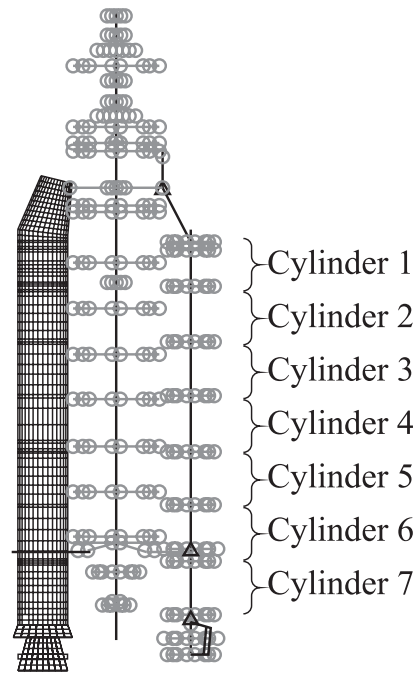

(c)

Figure 3 The SRB FE models in bench and flight configurations: (a) SRB beam model in test-bench (beam model) configuration; (b) SRB 3D volumetric model in testbench (3D super-element model) configuration; and (c) SRB beam and 3D volumetric models in flight configuration (beam model for central rocket body)

This long-lasting study was intended to analyze the thrust oscillation levels measured during the development and qualification phase (from 1993 to 1996), to characterize their expected maximum level and to prepare for the assessment of their impact on the launcher during flight. Different FE models have been built with various precision levels. Since the end of the development, these FE models have been used either for flight or test-bench exploitation or for further analyses, such as explanation of unexpected behavior (anomalies) or dynamic predictions in new configurations.

Two SRB models exist and can be used either in flight configuration or in test-bench configuration:

(1) the first model is constituted of beam elements ("beam model," see Fig. $3 a$ ): this modeling is very simple (around 300 degrees of freedom) but, nevertheless, representative in terms of mass and stiffness. The different constitutive elements of the SRB are meshed separately (domes, segments, cylinders, nozzle, etc.), in order to be easily adjustable to structural modifications like, for instance, the grain overloading of the front segment. The pressure excitation is applied as nodal forces on domes; and 


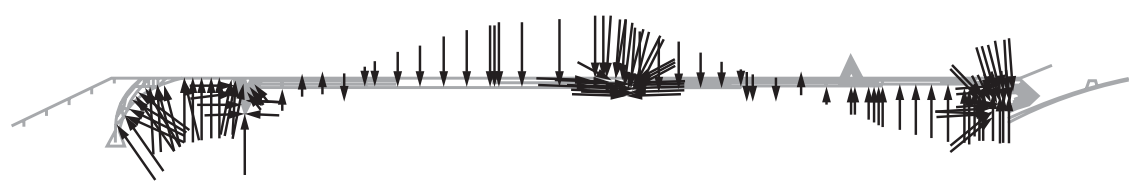

Figure 4 The 2nd acoustic pressure field inside the combustion chamber (3D volumetric model)

(2) the second model is constituted with shell, beam, and hexahedral elements (three-dimensional (3D) volumetric model, around 120000 degrees of freedom at ignition). This model is much finer than the beam model but logically less simple to be used and modified. The pressure inside the channel is directly applied on all the free surfaces. As a consequence, it is possible to apply a static pressure combined with a "half-sine" pressure wave (1st $\mathrm{AM})$ or a "sine" pressure wave (2nd AM) along the channel, like shown in Fig. 4.

\subsection{Simple Finite-Element Simulations with Beam Model}

Most of the dynamic-response simulations are performed with the SRB beam model, either in flight or in test-bench configuration. The range of accuracy of this kind of simulation is well known: these simulations, modal analyses, and harmonic responses are only reliable when global dynamic behavior is expected and when no resonance occurs.

The beam model is used for all flight analyses and explotations and allows reconstructing with an inverse method the 1st acoustic mode thrust oscillations. All the modal analyses are also performed with this model. Because of the simplicity to modify it associated with the reliability of the results, the FE beam model is a very useful and efficient tool.

\subsection{Accurate Finite-Element Simulations with Three-Dimensional Volumetric Model}

However, the SRB beam model is sometimes not accurate enough to simulate specific conditions such as resonance or local behavior. In that case, the SRB $3 \mathrm{D}$ volumetric model is used, because it offers high reliability, even in conditions like resonance where very accurate representativity is required.

Indeed, during one recent test-bench firing, very high vibration levels were measured on the case (such high levels had never been registered before), although the exciting pressure levels were quite low. First investigations led to 
suspect resonance conditions, which needed to be confirmed by measurement analyses and FE simulations with the $3 \mathrm{D}$ volumetric model. All the FE model evolutions and the performed simulations allowed, on the one hand, validating the model in test-bench configuration and, on the other hand, explaining and confirming an expected occurrence of resonance phenomenon: the motor has been excited by pressure oscillations almost on its 3rd yaw-bending eigenmode.

\subsubsection{Modal behavior}

Many eigenmodes can be tracked by measurement analysis. Some of them can be easily tracked just by computing sliding spectra on vibration signals (Fig. $5 \mathrm{a}$ ); for the others, it is necessary to use a more complicated method which allows catching at different times the frequency of the maximal transfer function be-

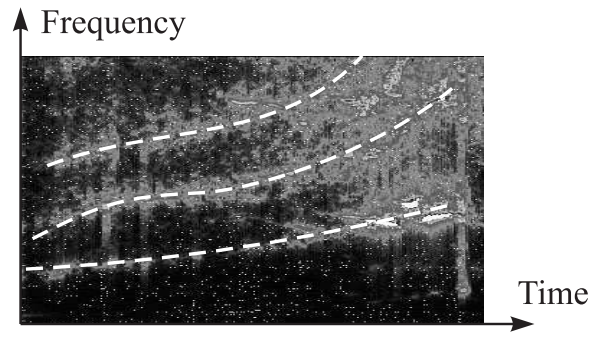

(a)

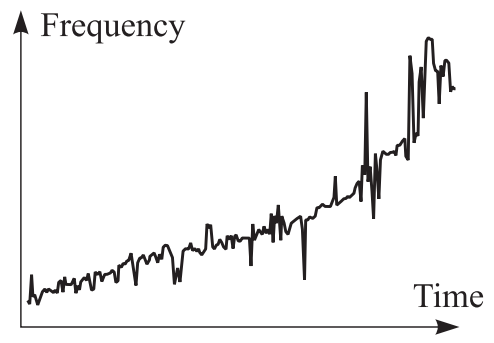

(b)

Figure 5 Mode tracking by measurement treatment: (a) sliding spectrum; and (b) sliding maximal transfer function

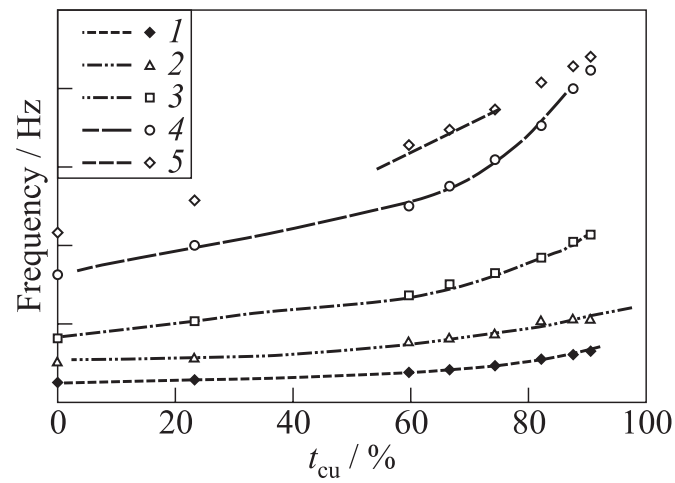

Figure 6 Experimental natural frequencies tracking (curves) vs. FE 3D volumetric modal computations (signs): numbers 1 to 5 refer to modes 1 to 5 
tween vibratory response and pressure excitation, even inside the time range and frequency range of pressure oscillations (Fig. $5 b$ ).

Due to the treatment of vibration measurements, it is possible to get the modal behavior of all excitable eigenmodes of the booster. Concurrently, the modal computation on the FE models (beam or 3D volumetric) allows tracking the numerical natural frequencies for all the modes at different combustion times. Since the comparison between experimental mode tracking and numerical computations provides excellent results, the FE 3D volumetric model in testbench configuration can be considered valid with respect to its modal behavior (Fig. 6).

\subsubsection{Harmonic responses}

\section{Description}

Even if the frequency of pressure oscillations is continuously shifting (see Fig. 2), the evolution is quite slow (around $0.1 \mathrm{~Hz} / \mathrm{s}$ ) compared to the phenomenon frequency. Thus, excitation by pressure oscillations can be considered harmonic.

The harmonic response simulation is very interesting when the $3 \mathrm{D}$ volumetric model is used, because this FE model can be loaded by physical pressure fields on internal free surfaces (see Fig. 4). To be representative of real pressure oscillations, the pressure field needs to have a good "shape" (meaning a good repartition along the channel) and the correct amplitude. Simulations have been performed on an already burned booster configuration in order to validate the model and the computation method (because many vibration measurements are available and can be used as reference for comparison). Moreover, the real amplitude of pressure oscillations measured at the front dome is used. Indeed, nowadays, there is no fully reliable computational tool available to predict the amplitude of pressure oscillations.

There are two different possibilities to define the pressure field of the harmonic pressure excitation. The easiest way is to assume that the pressure field has a perfect half-sine or complete sine shape (regarding the considered acoustic mode). However, as the real channel geometry is not exactly a cylinder, this approach may not be completely representative in local cavities between the two main segments or concerning the amplitude ratio between aft dome and front dome. That is why a modal acoustic computation is performed using an FE model of the cavity. It provides an acoustic field which is quite similar to the theoretical sine shape but which is supposed to be a bit more realistic (Fig. 7). First, the static uniform burning pressure is applied inside the entire channel. Then, the acoustic pressure field at the cavity/structure border is applied as an excitation on the structure internal free surfaces after having been scaled to the front dome measured amplitude. Rigid boundaries are used for this computation, 


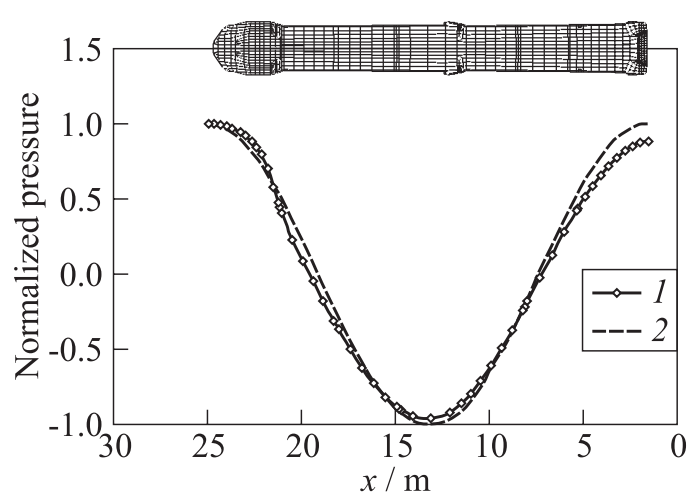

Figure 7 Normalized amplitude of acoustic pressure along the channel: 1 - computation; and 2 - theoretical sine

and the cavity mesh stops slightly upstream of the throat area (considering the acoustic impedance at the sonic throat). Some improvements of these simplifying hypotheses have been identified to increase the already satisfying accuracy of the computation.

Once the excitation has been defined and applied to the structure, the harmonic computation provides results for vibrations of the case, of the nozzle and associated to interfaces with the central rocket body, which are useful to define the load cases on the whole launcher.

\section{Example of Simulations}

In Fig. 2, the superposition of pressure excitation blasts on structural natural frequencies reveals that the booster is excited in resonance conditions. Moreover, the analysis of vibratory levels of this particular booster in bench configuration shows that they are much higher than those measured during former test-bench despite lower pressure excitation levels.

This typical resonance behavior appeared due to the shift of natural frequencies in the new configuration of the booster. As a matter of fact, the case cylinder junctions have been modified compared to the former design, decreasing the mass and the damping of the case without any major effect on the stiffness. As a consequence, the booster structural natural frequencies have been increased:

- appearance of a resonance on the 1 st $\mathrm{AM}$ (at about $82 \% t_{\mathrm{cu}}$ ); and

- modification of some crossing conditions with pressure oscillations sliding frequencies and excitation amplitude at resonance (2nd AM at about $69 \%$ $\left.t_{\mathrm{cu}}\right)$. 
These evolutions of the SRB dynamic behavior can typically be explained and also predicted by FE simulations.

The next paragraphs present the simulations performed for two particularly interesting blasts of the 1st and 2nd AM compared to available measurements.

1st $\mathrm{AM}$, around $82 \% t_{\mathrm{cu}}$ (normalized time):

Figure 8 shows the vibratory fields calculated with FE 3D model in comparison to experimental results at $82 \% t_{\text {cu }}$ for the excitation frequency of pressure oscillations. Even in quasi-resonance conditions, the computed vibration repartition along the case (labels of the cylinders available in Fig. $3 c$ ) is well representative of the real one. Such a harmonic FE simulation offers very good results, confirmed by the simulations of other blasts of the 1st and the 2nd AM.

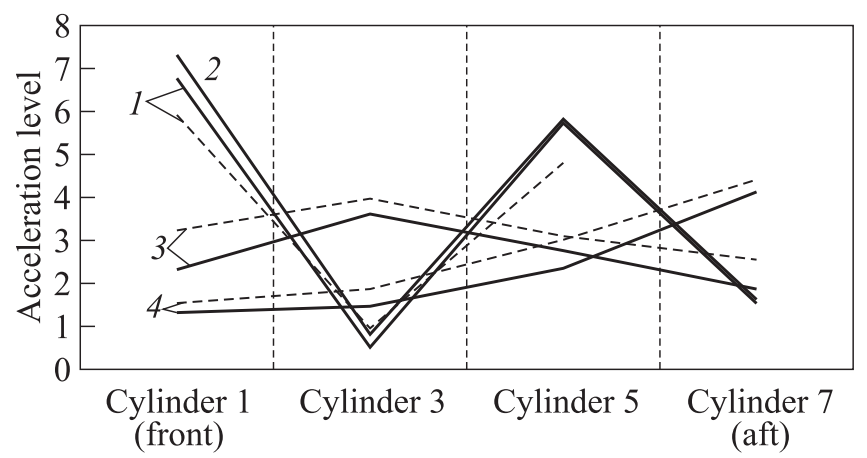

Figure 8 Measured (dashed lines) and simulated (solid lines) vibrations in yaw (1 G04 and $2-$ G16) and longitudinal (3- G04 and $4-$ G16) directions (1st AM blast 3)

2nd AM, around $69 \% t_{\mathrm{cu}}$ (normalized time):

Because of the modification of crossing conditions between pressure excitation and SRB longitudinal eigenmode (due to the case design evolution), the resonance on the 2nd blast of the 2 nd AM has become stronger and more severe. Pure resonance occurs earlier than with the former heavier design of the case (due to the shift of structural natural frequency), at a time with a higher induced pressure amplitude (Fig. 9). As a logical consequence, the maximal vibratory levels are higher.

The 3D simulations have been performed for the two case designs (the former and the new lightened one) at the measured pressure frequencies in test-bench configuration. Modal analysis first confirmed the frequency shift due to mass loss. Then, the harmonic response to acoustic excitation allowed simulating 


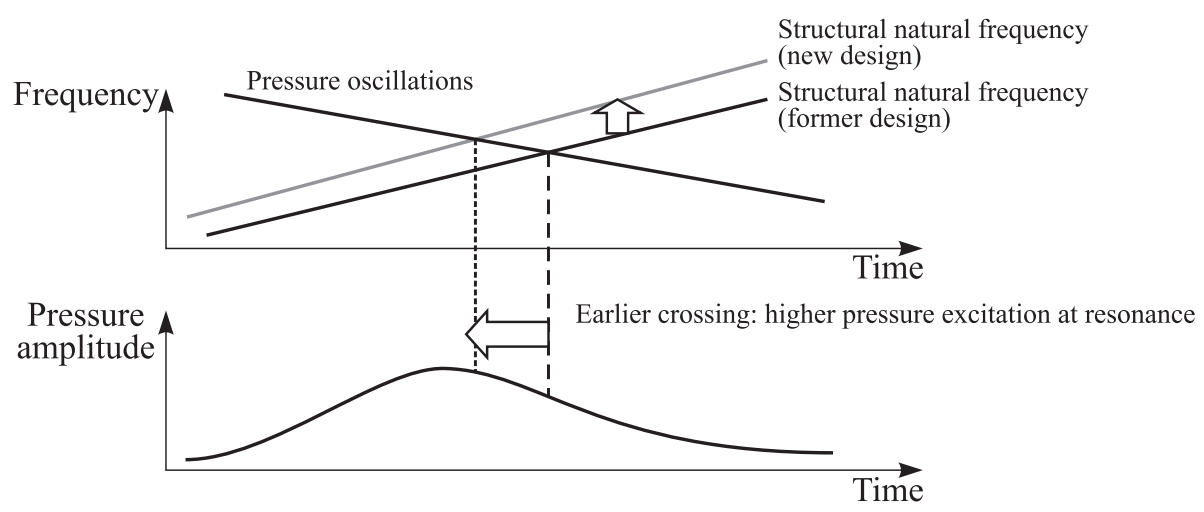

Figure 9 Resonance condition during 2 nd $\mathrm{AM}$ around $69 \% t_{\mathrm{cu}}$

correctly the dynamic behavior of the SRB excited by pressure oscillations at resonance: the calculated vibratory fields of the SRB case excited by the 2 nd acoustic mode pressure oscillations were similar to the measured fields.

\subsubsection{Finite-element model validation and further uses}

On the one hand, due to the modal validation and, on the other hand, due to the very reliable simulations of response to pressure oscillations (1st and 2 nd acoustic modes), even in sharp resonance conditions, the 3D volumetric FE model has been validated in test-bench configuration and is currently considered as reference. All tasks performed as a followup effort to simulate the dynamic behavior of the new lighter case design allowed understanding new resonance conditions (although the difference with the former configuration is minor) and to validate the FE model. It is henceforth available for further accurate previsions of dynamic behavior and enquiries in case of future potential anomalies in testbench configuration.

The validated FE $3 \mathrm{D}$ volumetric model is very useful to improve the use of the simpler FE beam model and the understanding of simulations performed with it. Indeed, it can be used to identify and associate the deformed shapes of the eigenmodes calculated with both $3 \mathrm{D}$ and beam models and to compare them with the results obtained from experimental measurements.

In flight configuration, the SRB booster is linked to the central rocket body through the DIAS (discoupling device), which is much more flexible than the front bench attachment. For this reason, the dynamic behavior of the SRB in this flight configuration is specific and different from the test-bench configuration. Confirmation of the $3 \mathrm{D}$ model validity for the test-bench configuration needs to be updated for the flight configuration. 


\section{FUTURE PROSPECTS}

\subsection{Improvement of the Acoustic Harmonic Excitation}

Modal acoustic pressure fields are calculated in a rigid undeformed cavity. Because in real burning conditions the cavity is deformed due to internal combustion pressure and according to excited structural eigenmodes, it would be preferable to calculate the acoustic modes in a deformed cavity. This would imply the creation of the new mesh to take into account pressure effects and/or to calculate simultaneously structural eigenmodes and acoustic eigenmodes for evaluating the respective effect of these two parameters.

\subsection{Validation of the Finite-Element Model in Flight Configuration}

Because of specific boundary conditions in the flight configuration, the FE 3D volumetric model cannot be directly used in the validated test-bench configuration. Some improvements have been identified to make it more representative of the real booster, in particular, concerning the discoupling device (DIAS) between the SRB and the central rocket body.

Once the FE model is modified, it will be necessary to validate it for the flight configuration in the same way as it was done for the test-bench configuration.

This flight-validated FE 3D volumetric model could be used for evaluating the new dynamic behavior induced by future design evolutions of the booster. It could be also used in the framework of anomalies: very accurate simulations could help understanding unexpected behavior observed during booster flights (transient phenomena at ignition, pressure oscillations, etc.).

Moreover, predictions of global dynamic environment generated by the SRB combustion could be performed with this FE model for the future design of the booster and then be used as an input for global system studies on the whole launcher.

\subsection{Transient Response Simulations}

There are many possibilities to define pressure loading in order to simulate the SRB behavior excited by pressure oscillations. The 3D model is loaded by physical acoustic pressure fields and has been validated with this kind of solicitation in test-bench configuration. Nevertheless, the performance of this excitation can be improved:

- acoustic pressure fields are calculated in the cavity, excluding some volume, notably the one close to the throat; 
- the static pressure which is applied in the channel is uniform;

- acoustic pressure fields are consistent with a simplified approach using a cylindrical geometry for the channel, but no data are available concerning the shape and the amplitude of the pressure field at the aft dome or in the middle-channel cavities to check the accuracy of applied pressure load; and

- the harmonic approach with acoustic pressure fields needs to simulate separately the 1st and 2nd AM excitations.

An alternative approach to harmonic computations has been developed to avoid some of the bias listed above: a transient response simulation. The aim is to simulate the dynamic response of the booster excited by pressure oscillations (all acoustic modes merged), with an expected better reliability for the cavity pressure field. Such a simulation has been developed in a Research and Technologies study within a CNES contract. The selected configuration was the flight configuration, because of the reliability of the available computed nonstationary pressure field, despite the fact that the FE 3D volumetric model had not yet been validated in that configuration.

This transient simulation allows using for the excitation a representative pressure field inside the channel (and no more uniform like in harmonic simulations), containing all harmonic acoustic components. Vibratory fields on the case and at interfaces between $\mathrm{SRB}$ and central rocket body are computed with the FE 3D model. Results are available as transient signals and are compared to experimental data, after specific frequency treatments. Figure 10 presents spectra of the calculated vibratory signal and measurements for cylinder 5 at the time chosen for the simulation (around $69 \% t_{\mathrm{cu}}$ ).

The performed transient simulations are very encouraging for this example: both vibration levels and peak frequencies (more or less for the 2nd AM) are

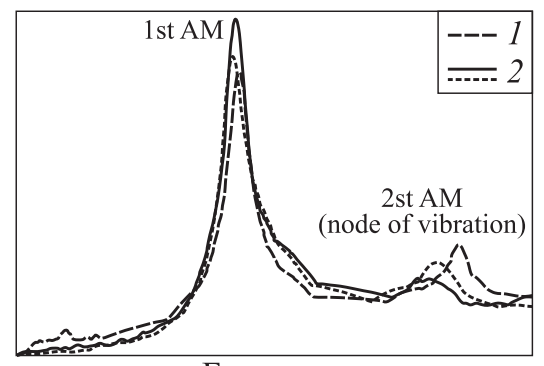

Frequency

Figure 10 Spectrum of calculated (1) and measured (2 and 3 - boosters 1 and 2, respectively) vibration signals 
representative of the flight measurement. Nevertheless, some imperfections still exist in this kind of simulation and need to be improved:

- it is necessary to calculate completely the nonstationary flow inside the cavity for each combustion time when pressure oscillations of any acoustic mode occur. Because these computations are very long, all blasts cannot be calculated;

- computed signals along the channel applied on internal free structure surfaces need to be very representative in terms of frequency; otherwise, they could modify real resonance conditions: a shift of $1 \mathrm{~Hz}$ on a 2nd AM frequency will dramatically reduce the reliability of dynamic simulations at resonance; and

- nowadays, totally reliable predictions of pressure oscillations are not possible: the above example was chosen because it was representative (for the 1st AM), but in many situations, the existing simulations do not provide enough accuracy (for instance, here, the frequency is not accurate enough for the 2nd AM).

\subsection{Dynamical Approach to Balance the Lack of Reliability of the Previsions of Pressure Excitation}

Various methods have been developed and validated to simulate the dynamic behavior of the SRB excited by pressure oscillations. In order to deal with the lack of reliability of pressure excitation previsions, it would be very useful to perform some sensitivity studies to identify the effect of the occurrence time and frequency of pressure oscillations (amplitude is not necessary because the FE models are linear) on the SRB dynamic behavior (transfer functions). Such analyses could indeed point out the critical excitation conditions in terms of dynamic behavior and calculate the associated most critical dynamic response for a normalized pressure amplitude. Nevertheless, the amplitudes of pressure oscillations are necessary to quantitatively predict vibration levels.

\section{CONCLUDING REMARKS}

Many tools and simulation methods have been developed by Snecma Propulsion Solide (Safran Group) for the account of Europropulsion and CNES in order to simulate correctly the dynamic behavior of the SRB excited by internal pressure oscillations.

Different FE models are available depending on the accuracy required for analysis (beam model, 3D volumetric model) in flight or test-bench configuration. 
Some improvements of the finer 3D model need to be achieved to complete its validation in flight configuration, already acquired in test-bench configuration.

Harmonic responses are performed after a modal acoustic pressure field computation, which is used afterwards as the excitation for the structural FE model. Even if all the identified improvements have not been implemented yet, this simulation is sensitive to slight evolutions of design and provides very good results, even in resonance conditions.

In order to improve the representativity of the pressure excitation, a transient method has been developed. The pressure field is obtained from an nonstationary flow computation: it should allow a more physical pressure repartition inside the cavity than with the acoustic computation. Moreover, excitation signals also contain all acoustic harmonic components. Results are very encouraging but are very sensitive to the precision of the pressure excitation.

Validated FE simulations (both harmonic and transient) of the SRB dynamic behavior could be very useful in future dynamic studies of the whole launcher: the SRB dynamic behavior would be simulated with the FE 3D model in the validated flight configuration (with the simplified central rocket body FE beam model), and the induced dynamic environment computed at interfaces with the central rocket body would be an input for global system studies for the whole launcher performed by the launcher architect. 